\title{
Cancer Type and Risk of Newly Diagnosed Depression Among Elderly Medicare Beneficiaries With Incident Breast, Colorectal, and Prostate Cancers
}

\author{
Monira Alwhaibi, $\mathrm{PhD}^{\mathrm{a}, \mathrm{b}}$; Usha Sambamoorthi, $\mathrm{PhD}^{\mathrm{a}}$; Suresh Madhavan, $\mathrm{PhD}^{\mathrm{a}}$; Thomas Bias, $\mathrm{PhD}^{\mathrm{c}}$; \\ Kimberly Kelly, $\mathrm{PhD}^{\mathrm{a}}$; and James Walkup, $\mathrm{PhD}^{\mathrm{d}}$
}

\begin{abstract}
Background: Elderly individuals (age $>65$ years) with cancer are at high risk for newly diagnosed depression after a cancer diagnosis. It is not known whether the risk of newly diagnosed depression varies by cancer type. Purpose: To examine the variations in the risk of newly diagnosed depression by cancer type among elderly individuals with cancer. Methods: This study used a retrospective cohort study design and data from the linked SEER-Medicare files. Elderly individuals (age $>65$ years) with incident breast, colorectal (CRC), and prostate cancers diagnosed between 2007 and 2011 ( $N=53,821)$ were followed for 12 months after cancer diagnosis. Depression diagnosis was identified during the 12-month follow-up period after cancer diagnosis using the ICD-9-Clinical Modification. Complementary log-log regression was used to examine the association between cancer type and risk of newly diagnosed depression after adjusting for other risk factors for depression. Results: We found a significantly higher percentage of newly diagnosed depression among women with CRC compared with those with breast cancer (5.8\% vs 3.9\%), and among men with CRC compared with those with prostate cancer (3.4\% vs $1.6 \%$ ). In the adjusted analysis, women with CRC had a $28.0 \%$ higher risk of newly diagnosed depression compared with women with breast cancer (adjusted risk ratio [ARR], 1.28; $95 \%$ CI, 1.12-1.46) and men with CRC had a 104.0\% higher risk of newly diagnosed depression compared with those with prostate cancer (ARR, 2.04; $95 \% \mathrm{Cl}, 1.65-2.51)$. Conclusions: Our findings identified cancer types associated with a high risk of newly diagnosed depression after cancer diagnosis, who might benefit from routine depression screening to help in its early detection and treatment.
\end{abstract}

J Natl Compr Canc Netw 2017;15(1):46-55

\section{Background}

Depression is a highly prevalent mental health condition among elderly cancer survivors (age $>65$ years). According to the CDC, "cancer survivor refers to a person who has been diagnosed with cancer, from the time of diagnosis throughout his or her life." Cancer survivors often find the diagnosis of cancer to be a very stressful life event. They may face a fear of death; changes in physical health, life plans, work, and social roles; and some financial concerns. ${ }^{2}$ As a result, some cancer survivors

From a Department of Pharmaceutical Systems and Policy, School of Pharmacy, West Virginia University, Morgantown, West Virginia;

'Department of Clinical Pharmacy, School of Pharmacy, King Saud University, Saudi Arabia; 'Department of Health Policy, Management, and Leadership,

School of Public Health, West Virginia University, Morgantown, West Virginia; and ${ }^{\mathrm{D} C l i n i c a l}$ Psychology Department, Graduate School of Applied and Professional Psychology, Rutgers University, New Brunswick, New Jersey. Submitted April 26, 2016; accepted for publication September 7, 2016.

The authors have disclosed that they have no financial interests,

arrangements, affiliations, or commercial interests with the manufacturers may have difficulties adjusting to the diagnosis of cancer and may develop psychological side effects. Evidence from the literature suggests that the psychological effects of cancer diagnosis may range from sadness to depressive symptoms to clinical depression, hereinafter referred to as "depression." ${ }^{3}$ Depression is highly prevalent among cancer survivors compared with their matched noncancer controls. ${ }^{4-6}$ It is estimated that $8 \%$ to $25 \%$ of all cancer survivors experience depression; specifically, that $10 \%$ to $25 \%$ of breast cancer survivors, $5,7,8 \%$ to $18 \%$

of any products discussed in this article or their competitors.

This research project was supported by a grant from the Research Center of the Center for Female Scientific and Medical Colleges, Deanship of Scientific Research, King Saud University.

Author Contributions: Data analysis: Alwhaibi, Sambamoorthi. Interpretation of data and manuscript preparation: Alwhaibi, Sambamoorthi, Madhavan, Bias, Kelly, Walkup.

Correspondence: Monira Alwhaibi, PhD, West Virginia University, School of Pharmacy, PO Box 9510, Morgantown, WV 26506.

E-mail:mmalwhaibi@mix.wvu.edu; dr.alwhaibi@gmail.com 
of colorectal cancer (CRC) survivors, ${ }^{9,10}$ and $5 \%$ to $10 \%$ of prostate cancer survivors will experience depression. ${ }^{11,12}$

Variability in the prevalence rates of depression may be due to heterogeneous samples and/or differences in settings, time periods, and instruments that are used to diagnose depression. Studies based on claims databases use the International Classification of Diseases, Ninth Revision, Clinical Modification (ICD-9-CM) codes to identify depression. ${ }^{9-12}$ These studies reported the lowest prevalence rates, followed by studies using the Diagnostic and Statistical Manual of Mental Disorders, Fifth Edition (DSM-V) structured interview. ${ }^{7}$ Studies that use the self-reported symptoms scales, such as the Beck Depression Inventory, with cutoff points to diagnose depression have reported the highest prevalence rates of depression. ${ }^{7,8}$ For example, the prevalence rate of depression among women with breast cancer was $6 \%$ based on ICD-9-CM codes, ${ }^{5}$ $11 \%$ (95\% CI, $8 \%-16 \%$ ) based on diagnostic interviews, and 20\% (95\% CI, 16\%-24\%) based on self-reported symptoms scale. ${ }^{13}$

Furthermore, the risk of newly diagnosed depression can be higher among cancer survivors compared with age- and sex-matched noncancer cohorts. A retrospective cohort study estimated that the incidence rate of depression among women with breast cancer was $2.4 \%$, and that women with breast cancer had a $58 \%$ higher risk of newly diagnosed depression within a year after cancer diagnosis compared with their matched noncancer counterparts (adjusted risk ratio [ARR], 1.58; 95\% CI, 0.84-3.0)..$^{5}$

There are many reasons why patients with cancer may be at a higher risk of developing depression compared with those without cancer. The cancer diagnosis itself may lead to depression. For example, studies found that individuals diagnosed at an advanced stage have a higher risk of developing depression compared with those diagnosed at an early stage. 5,14,15 Cancer treatment can also affect the risk of developing depression. Retrospective studies among women with breast cancer reported a significant association between chemotherapy treatment and the risk of depression. ${ }^{16-18}$ Among men with prostate cancer, those who received androgen deprivation therapy or radiotherapy had a higher risk of depression compared with those who underwent surgery. ${ }^{19-22}$ Cancer-related physical symptoms such as pain, fatigue, and other physical limitations may lead to depression. Cancer survivors who reported fatigue or pain had higher rates of depression than those who did not report fatigue or pain. ${ }^{23-25}$

Moreover, the risk of newly diagnosed depression may be higher among some cancer types compared with others due to differences in survival prognosis and stage at diagnosis. ${ }^{5,26}$ The estimated 5 -year relative survival rate is $65 \%$ for individuals with CRC compared with $89 \%$ for women with breast cancer and $99 \%$ for men with prostate cancer. ${ }^{26}$ Danese et $\mathrm{al}^{5}$ reported that elderly women with breast cancer diagnosed at an advanced stage (stage IV) had a higher risk of newly diagnosed depression compared with those diagnosed at an early stage (stage I) (risk ratio [RR], 5.03; 95\% CI, 3.45-7.35). CRC is more likely to be diagnosed at an advanced stage and to have a poor survival prognosis compared with breast and prostate cancers. ${ }^{5,26}$ Therefore, patients with CRC may have a higher risk of newly diagnosed depression compared with those with breast or prostate cancers.

Although the risk of newly diagnosed depression may vary by cancer type, there is a paucity of research on the variations in the risk of newly diagnosed depression by cancer type. Identifying cancer survivors, who are at high risk of newly diagnosed depression, is important because depression can negatively affect health-related quality of life ${ }^{27}$ and survival after cancer diagnosis. ${ }^{9,11,27}$ Depression among cancer survivors is also associated with high healthcare utilization and expenditures compared with their counterparts without depression. ${ }^{11,28-30}$ For example, elderly patients with both cancer and depression were more likely to have emergency room visits, inpatient visits, outpatient visits, readmission, and medication use compared with those without depression. ${ }^{11,28,29}$ Therefore, the objective of this study was to examine variations in the risk of newly diagnosed depression among the most common cancer types, ${ }^{26}$ with the following comparisons: women with breast cancer compared with women with CRC, and men with prostate cancer compared with men with CRC. These cancers were selected because they are the most common cancer types; breast cancer among women $(41 \%)$, prostate cancer among men (45\%), and CRC among men and women $(8 \%) .{ }^{26}$ 


\section{Conceptual Framework}

This study adapted the determinants-of-health model by Marmot and Wilkinson, ${ }^{31}$ which posits that many dimensions affect an individual's health or disease/ illness (eg, newly diagnosed depression). These dimensions are an individual's physical make-up, social support, access to care, health behaviors, psychological factors, biological risk factors, treatment factors, community resources, and geographic region.

\section{Methods}

\section{Data Source}

SEER-Medicare: This study used the linked SEERMedicare files. The SEER Program is an epidemiologic surveillance system consisting of population-based tumor registries residing in 18 SEER areas. ${ }^{32}$ SEER cancer registries collect data on all incident cases of cancer that occur in persons residing in SEER areas. These data are available in the Patient Entitlement and Diagnosis Summary File (PEDSF), which has information on patient demographic characteristics, cancer type, tumor characteristics, and chemotherapy and radiation therapy provided within 4 months of cancer diagnosis. Because Medicare is the primary health insurer for the elderly, SEER data have been linked to Medicare claims. Medicare claims files consist of inpatient claims (Medicare Provider Analysis and Review), outpatient claims (National Claims History files and outpatient claims files), and prescription drug file.

\section{American Community Survey Estimates From}

Census: This study used the American Community Survey (ACS) 2008 to 2012 census tract and census zip code files. We linked these files to PEDSF files by geographic codes, which included state and county. We used these files to derive the census tract median household income and education level.

The Area Health Resource File: The Area Health Resource File (AHRF) is a publicly available data file provided by the Department of Health \& Human Services (DHHS), which includes county, state, and national files. ${ }^{33}$ This study used the AHRF to provide county-level urban/rural continuum codes, the presence of community mental health clinics (CMHCs), and health professional shortage area of mental healthcare. We linked the AHRF files to PEDSF files by geographic codes, which included state and county.

\section{Study Design}

This study used a retrospective cohort study design with a baseline and follow-up period. We considered the date of cancer diagnosis as the index date. We defined the 12 months before the index date as the baseline period and the 12 months after the index date as the follow-up period.

\section{Study Population}

Identification of Cancer Survivors: The study population consisted of elderly patients (age $>65$ years) with cancer who were diagnosed with primary-only incident breast, CRC, and prostate cancers between 2007 and 2011. We imposed the age restriction of $>65$ years to allow for a 12 -month baseline period to identify chronic conditions and other risk factors before cancer diagnosis. We identified the cancer types using the primary site variable and the ICD-Oncology, Third Edition (ICD-O-3) histology codes.

\section{Depression-Free Individuals at Cancer Diagnosis:}

We identified a depression-free cohort among individuals with incident cancer. To ensure that the incident cancer cases were depression-free, we used validated criteria from the National Committee for Quality Assurance (NCQA). ${ }^{34}$ According to the NCQA, individuals are considered to be depressionfree at index date if they did not receive antidepressants 90 days before the index date (ie, date of cancer diagnosis) or did not have a depression diagnosis 120 days before the index date.

Other Inclusion/Exclusion Criteria: We required that all individuals have continuous enrollment in Medicare Parts A and B and no enrollment in managed care plans during the baseline and follow-up periods. To identify antidepressants 90 days before the index date, we also required that individuals have continuous enrollment in Medicare Part D for 3 months before cancer diagnosis. We excluded individuals with an unknown stage at diagnosis, diagnosed through autopsy or death certificate, and who died during the follow-up period of 12 months. Supplemental eAppendix 1 illustrates the analytical population selection process (available with this article at JNCCN.org).

\section{Dependent Variable: Newly Diagnosed Depression (Yes/No)}

The dependent variable was newly diagnosed depression after cancer diagnosis (newly diagnosed depression) in breast, CRC, and prostate cancer survivors. 
To identify depression diagnosis, we used a validated algorithm developed by the CMS Chronic Conditions Data Warehouse. ${ }^{35}$ Individuals with at least one inpatient or outpatient visit with a depression diagnosis during the 12-month follow-up period were classified as having newly diagnosed depression. We identified the depression diagnosis using ICD-9-CM codes, which included 296.2 (major depressive disorder, single episode), 296.3 (major depressive disorder, recurrent episode), 298.0 (depressive type psychosis), 300.4 (neurotic depression), 309.1 (prolonged depressive reaction), and 311.0 (depressive disorder, not classified). These codes were widely used in previously published studies to identify depression diagnosis among Medicare beneficiaries. ${ }^{11,36,37}$

\section{Key Independent Variable}

We selected the independent variables based on the determinants-of-health model. The key independent variable was cancer type, which is considered a biological risk factor. Cancer types were women with breast cancer, women with CRC, men with CRC, and men with prostate cancer.

\section{Other Independent Variables}

Individual physical makeup variables included age in years at cancer diagnosis and race. Social support included marital status. Access to care was measured by primary care physician (PCP) visit quartiles, census tract median household income quartiles, and percentage with a less than high school education level quartiles. Health behavior consisted of tobacco use. Psychological factors included the presence of anxiety. Biological risk factors were cancer stage at diagnosis (based on the AJCC) and chronic physical conditions. Chronic conditions categories included cardiovascular disease (heart disease, diabetes, hyperlipidemia, hypertension, stroke), respiratory disease (asthma, chronic obstructive pulmonary disease), and musculoskeletal disease (arthritis, osteoporosis). These conditions were selected based on the list of chronic conditions framework developed by DHHS Multiple Chronic Conditions working group for research, planning, programs, and policy purposes. ${ }^{38}$ We identified these conditions based on a validated algorithm developed by the CMS Chronic Conditions Data Warehouse ${ }^{35}$ : individuals had at least 1 inpatient or outpatient visit during the baseline period. Treatment factors included cancer treatment during the 6 months after cancer diagnosis, which included chemotherapy, radiation therapy, and surgery. Cancer treatment was identified from claims data using the ICD-9-CM Healthcare Common Procedure Coding System (HCPCS) and the Common Procedural Terminology (CPT) codes. Community resources consisted of the presence or absence of county-level CMHCs and whether the county of residence was designated as a health professional shortage area for mental health. Geographical location consisted of the SEER region and county metropolitan status, which was defined using 2013 urban/rural continuum codes from the US Department of Agriculture's Economic Research Service. To control for changes in patterns of diagnosis over time, year of cancer diagnosis was also included as an independent variable.

\section{Statistical Analysis}

We used chi-square tests to test the significance of unadjusted differences in baseline characteristics and newly diagnosed depression. We used complementary log-log regression analysis to examine the adjusted associations between cancer types and risk of newly diagnosed depression with 4 different models. In Model 1, we included only cancer types without controlling for other factors. In Model 2 , we controlled for individual physical make-up (eg, age, race) and social support. In Model 3, we additionally controlled for access to care, health behaviors, biological risk factors (stage at cancer diagnosis and chronic physical conditions), and psychological factors. In Model 4, we controlled for cancer treatment factors, county-level community resources, geographic region, the year at cancer diagnosis, and all other risk factors in Model 3 (age, race, marital status, PCP visits, median household income, education level, tobacco use, anxiety, cancer severity measured by stage at diagnosis, and chronic physical conditions). All statistical analyses were carried out using SAS 9.4 (SAS Institute Inc., Cary, NC).

\section{Results}

\section{Characteristics of the Study Population}

The study population consisted of 53,821 elderly fee-for-service Medicare beneficiaries with incident breast, CRC, or prostate cancer who were depression-free at the time of cancer diagnosis. In the 
Alwhaibi et al

Table 1. Selected Characteristics of the Study Population by Cancer Type ${ }^{a}$

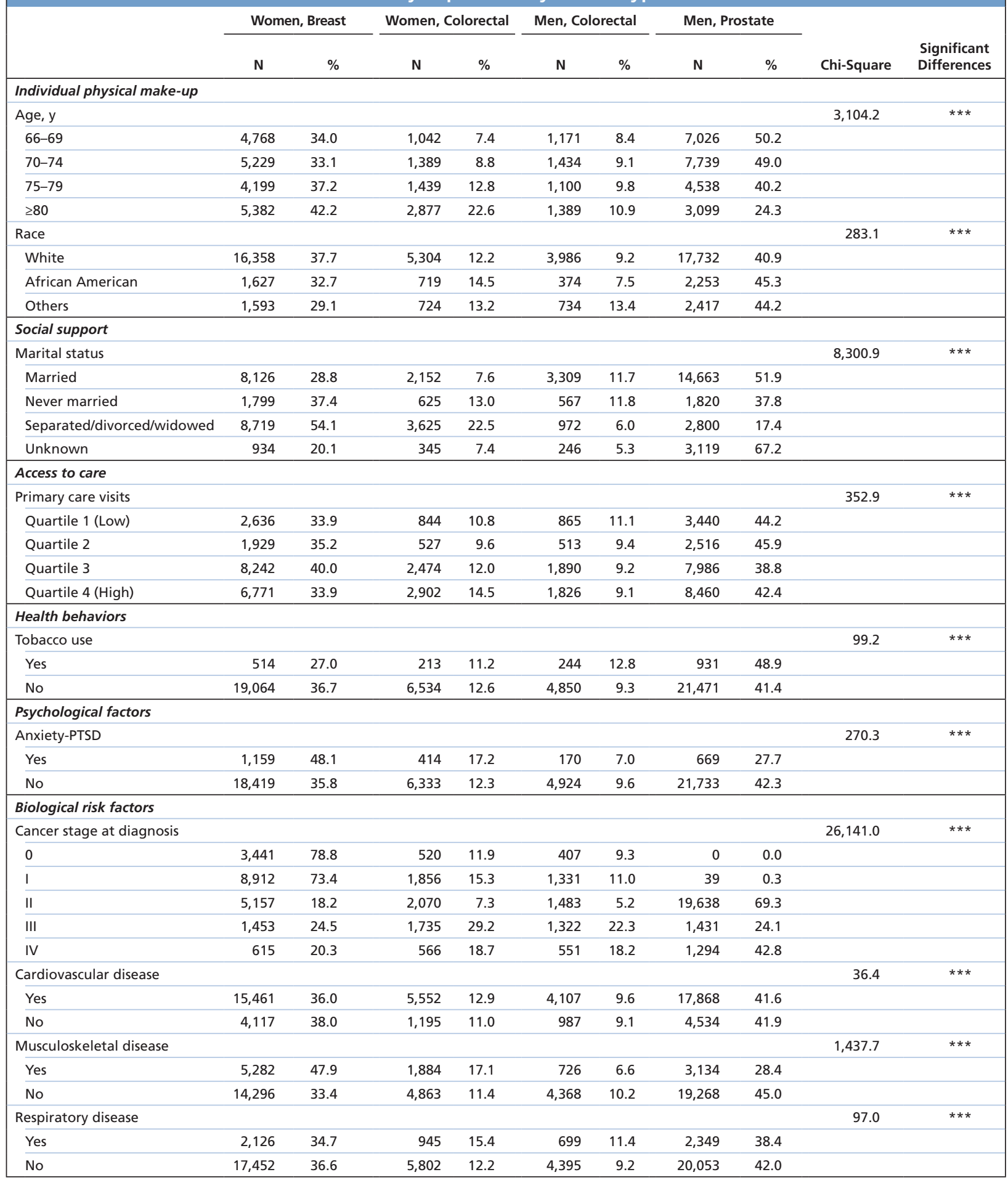

(continued on next page)

Abbreviations: CMHC, Community Mental Health Center; HPSA, health professional shortage area; PTSD, posttraumatic stress disorder.

aBased on 53,821 elderly fee-for-service Medicare beneficiaries with incident breast, colorectal, and prostate cancers who were continuously enrolled in

Medicare Parts A and B during the observation period and who were alive during the observation period. Asterisks represent significant differences in study population characteristics by breast, colorectal, and prostate cancer, derived from chi-square statistics.

${ }^{*} .01 \leq P<.05 ;{ }^{* *} .001 \leq P<.01 ; * * * P<.001$. 
Risk of Depression and Cancer Type

\begin{tabular}{|c|c|c|c|c|c|c|c|c|c|c|}
\hline & \multicolumn{2}{|c|}{ Women, Breast } & \multicolumn{2}{|c|}{ Women, Colorectal } & \multicolumn{2}{|c|}{ Men, Colorectal } & \multicolumn{2}{|c|}{ Men, Prostate } & \multirow[b]{2}{*}{ Chi-Square } & \multirow{2}{*}{$\begin{array}{l}\text { Significant } \\
\text { Differences }\end{array}$} \\
\hline & $\mathbf{N}$ & $\%$ & $\mathrm{~N}$ & $\%$ & $\mathrm{~N}$ & $\%$ & $\mathrm{~N}$ & $\%$ & & \\
\hline \multicolumn{11}{|l|}{ Treatment factors } \\
\hline Chemotherapy & & & & & & & & & $1,028.3$ & $* * *$ \\
\hline Yes & 3,884 & 26.6 & 1,674 & 11.4 & 1,601 & 10.9 & 7,469 & 51.1 & & \\
\hline No & 15,694 & 40.0 & 5,073 & 12.9 & 3,493 & 8.9 & 14,933 & 38.1 & & \\
\hline Radiation therapy & & & & & & & & & $4,610.0$ & $* * *$ \\
\hline Yes & 9,193 & 46.0 & 600 & 3.0 & 686 & 3.4 & 9,517 & 47.6 & & \\
\hline No & 10,385 & 30.7 & 6,147 & 18.2 & 4,408 & 13.0 & 12,885 & 38.1 & & \\
\hline Surgery & & & & & & & & & $23,164.3$ & $* * *$ \\
\hline Yes & 18,193 & 53.8 & 5,779 & 17.1 & 4,131 & 12.2 & 5,723 & 16.9 & & \\
\hline No & 1,385 & 6.9 & 968 & 4.8 & 963 & 4.8 & 16,679 & 83.4 & & \\
\hline \multicolumn{11}{|l|}{ Community resources } \\
\hline $\mathrm{CMHC}$ & & & & & & & & & 5.5 & \\
\hline Yes & 10,030 & 36.5 & 3,496 & 12.7 & 2,648 & 9.6 & 11,332 & 41.2 & & \\
\hline No & 9,548 & 36.3 & 3,251 & 12.4 & 2,446 & 9.3 & 11,070 & 42.1 & & \\
\hline HPSA & & & & & & & & & 57.3 & $* * *$ \\
\hline No shortage & 1,871 & 36.7 & 677 & 13.3 & 477 & 9.4 & 2,075 & 40.7 & & \\
\hline Whole county & 9,693 & 35.3 & 3,319 & 12.1 & 2,736 & 10.0 & 11,700 & 42.6 & & \\
\hline Part county & 8,014 & 37.7 & 2,751 & 12.9 & 1,881 & 8.8 & 8,627 & 40.6 & & \\
\hline \multicolumn{11}{|l|}{ Geographic location } \\
\hline Region & & & & & & & & & 181.4 & $* * *$ \\
\hline Northeast & 3,852 & 37.7 & 1,515 & 14.8 & 942 & 9.2 & 3,919 & 38.3 & & \\
\hline South & 5,003 & 36.9 & 1,733 & 12.8 & 1,312 & 9.7 & 5,516 & 40.7 & & \\
\hline North-central & 2,603 & 36.8 & 987 & 13.9 & 627 & 8.9 & 2,863 & 40.4 & & \\
\hline West & 8,120 & 35.4 & 2,512 & 10.9 & 2,213 & 9.6 & 10,104 & 44.0 & & \\
\hline Metropolitan status & & & & & & & & & 9.2 & * \\
\hline Metropolitan county & 15,947 & 36.5 & 5,460 & 12.5 & 4,056 & 9.3 & 18,196 & 41.7 & & \\
\hline Non-metropolitan county & 3,631 & 35.7 & 1,287 & 12.7 & 1,038 & 10.2 & 4,206 & 41.4 & & \\
\hline Year at cancer diagnosis year & & & & & & & & & 31,901 & ** \\
\hline 2007 & 2,876 & 34.9 & 1,068 & 13.0 & 797 & 9.7 & 3,495 & 42.4 & & \\
\hline 2008 & 3,916 & 35.2 & 1,433 & 12.9 & 1,110 & 10.0 & 4,655 & 41.9 & & \\
\hline 2009 & 4,091 & 36.6 & 1,443 & 12.9 & 1,006 & 9.0 & 4,627 & 41.4 & & \\
\hline 2010 & 4,208 & 37.2 & 1,372 & 12.1 & 1,065 & 9.4 & 4,666 & 41.3 & & \\
\hline 2011 & 4,487 & 37.4 & 1,431 & 11.9 & 1,116 & 9.3 & 4,959 & 41.3 & & \\
\hline
\end{tabular}

Abbreviations: CMHC, Community Mental Health Center; HPSA, health professional shortage area; PTSD, posttraumatic stress disorder.

a Based on 53,821 elderly fee-for-service Medicare beneficiaries with incident breast, colorectal, and prostate cancers who were continuously enrolled in Medicare Parts A and B during the observation period and who were alive during the observation period. Asterisks represent significant differences in study population characteristics by breast, colorectal, and prostate cancer, derived from chi-square statistics.

${ }^{*} .01 \leq P<.05 ; * * .001 \leq P<.01 ; * * * P<.001$.

study population, $36.4 \%$ were women with breast cancer, $12.5 \%$ were women with CRC, $9.5 \%$ were men with CRC, and $41.6 \%$ were men with prostate cancer (data not shown in tabular form). Most of the study population was white $(80.7 \%)$ and lived in metropolitan areas $(81 \%)$. We also found that $51.0 \%$ of the counties had a shortage of mental health professionals and $48.9 \%$ did not have CMHCs (Table 1).

\section{Cancer Types and Newly Diagnosed Depression}

Overall, $3.3 \%$ of elderly Medicare patients with breast, $\mathrm{CRC}$, and prostate cancer had newly diagnosed de- pression during the follow-up year. A chi-square analysis showed that newly diagnosed depression rates significantly differed by cancer type $(P<.001)$ (Table 2$)$. We found a significantly higher percentage of newly diagnosed depression among women with CRC compared with those with breast cancer $(5.8 \%$ vs $3.9 \%)$, and among men with CRC compared with those with prostate cancer (3.4\% vs $1.6 \%)$.

Table 3 displays the RRs and ARR of newly diagnosed depression by cancer types from multivariable complementary log-log regression analyses. In Model 1, which included only cancer types, women with CRC had a $53 \%$ higher risk of newly diagnosed depression 
(RR, 1.53; 95\% CI, 1.36-1.73) compared with women with breast cancer; men with CRC had a $111 \%$ higher risk of newly diagnosed depression (ARR, 2.12; 95\% CI, 1.74-2.57) compared with men with prostate cancer. This association between cancer type and risk of newly diagnosed depression persisted in Models 2 to 4 , even after controlling for all the risk factors and the year at cancer diagnosis in the final model.

\section{Sex and Newly Diagnosed Depression}

We found a significantly higher percentage of newly diagnosed depression among women with CRC compared with men with CRC (5.8\% vs $3.4 \%)$. In the adjusted analyses, we found that women with CRC had a $46 \%$ higher risk of newly diagnosed depression compared with men with CRC (ARR, 1.46; 95\% CI, 1.22-1.76) (Table 4).

\section{Cancer Stage and Newly Diagnosed Depression}

We found that the risk of newly diagnosed depression was higher among cancer survivors diag- nosed at an advanced stage compared with those diagnosed at an early stage. For example, cancer survivors diagnosed at stage IV had a $63 \%$ higher risk of newly diagnosed depression compared with those diagnosed at stage I (ARR, 1.63; 95\% CI, 1.31-2.03). The RR and ARR of newly diagnosed depression by other variables are displayed in supplemental eAppendix 2.

\section{Discussion}

This is the first study to date that has evaluated the relationship between cancer type and risk of newly diagnosed depression after cancer diagnosis among elderly individuals with incident breast, CRC, and prostate cancer. In our study, the rate of newly diagnosed depression was highest $(5.8 \%)$ among women with CRC and lowest (1.6\%) among men with prostate cancer. Among women with breast cancer the rate of newly diagnosed depression was $3.9 \%$ in our study, which is higher than the estimate $(2.4 \%)$

\begin{tabular}{|c|c|c|c|c|c|c|}
\hline & \multicolumn{2}{|c|}{ Depression } & \multicolumn{2}{|c|}{$\begin{array}{c}\text { No } \\
\text { Depression }\end{array}$} & \multirow[b]{2}{*}{ Chi-Square } & \multirow[b]{2}{*}{ Significant Differences } \\
\hline & $\mathbf{N}$ & $\%$ & $\mathbf{N}$ & $\%$ & & \\
\hline Cancer types & & & & & 365.7 & * \\
\hline Women, breast & 755 & 3.9 & 18,823 & 96.1 & & \\
\hline Women, colorectal & 394 & 5.8 & 6,353 & 94.2 & & \\
\hline Men, colorectal & 173 & 3.4 & 4,921 & 98.6 & & \\
\hline Men, prostate & 364 & 1.6 & 22,038 & 98.4 & & \\
\hline Stage at cancer diagnosis & & & & & 95.8 & * \\
\hline 0 & 154 & 3.5 & 4,214 & 96.5 & & \\
\hline 1 & 433 & 3.6 & 11,705 & 96.4 & & \\
\hline II & 705 & 2.5 & 27,643 & 97.5 & & \\
\hline III & 262 & 4.4 & 5,679 & 95.6 & & \\
\hline IV & 132 & 4.4 & 2,894 & 95.6 & & \\
\hline Chemotherapy & & & & & 0.1 & \\
\hline Yes & 454 & 3.1 & 14,174 & 96.9 & & \\
\hline No & 1,232 & 3.1 & 37,961 & 96.9 & & \\
\hline Radiation therapy & & & & & 49.5 & * \\
\hline Yes & 489 & 2.4 & 19,507 & 97.6 & & \\
\hline No & 1,197 & 3.5 & 32,628 & 96.5 & & \\
\hline Surgery & & & & & 110.6 & * \\
\hline Yes & 1,265 & 3.7 & 32,561 & 96.3 & & \\
\hline No & 421 & 2.1 & 19,574 & 97.9 & & \\
\hline
\end{tabular}

aBased on 53,821 elderly fee-for-service Medicare beneficiaries with incident breast, colorectal, and prostate cancers who were continuously enrolled in Medicare Part A and B during the observation period and who were alive during the observation period. Asterisks represent significant differences in newly diagnosed depression by cancer types, derived from chi-square tests.

$* P<.001$. 
Risk of Depression and Cancer Type

Table 3. ARRs and $95 \%$ Cls of Cancer Types From Regression Analysis on Newly Diagnosed Depressiona

\begin{tabular}{|c|c|c|c|c|c|c|c|}
\hline Cancer Types ${ }^{b}$ & RR & $95 \% \mathrm{Cl}$ & $\begin{array}{l}\text { Significant } \\
\text { Differences }\end{array}$ & Cancer Types ${ }^{c}$ & RR & $95 \% \mathrm{Cl}$ & $\begin{array}{l}\text { Significant } \\
\text { Differences }\end{array}$ \\
\hline \multicolumn{8}{|c|}{ Model 1: Included the types of cancer without adjustment } \\
\hline Women, colorectal & 1.53 & {$[1.36,1.73]$} & * & Women, breast & 2.29 & {$[1.97,2.67]$} & * \\
\hline Men, colorectal & 0.88 & {$[0.74,1.04]$} & & Women, colorectal & 3.10 & {$[2.62,3.66]$} & * \\
\hline Men, prostate & 0.42 & {$[0.37,0.47]$} & * & Men, colorectal & 2.12 & {$[1.74,2.57]$} & * \\
\hline Cancer Types ${ }^{b}$ & ARR & $95 \% \mathrm{Cl}$ & $\begin{array}{l}\text { Significant } \\
\text { Differences }\end{array}$ & Cancer Types ${ }^{c}$ & ARR & $95 \% \mathrm{Cl}$ & $\begin{array}{c}\text { Significant } \\
\text { Differences }\end{array}$ \\
\hline \multicolumn{8}{|c|}{ Model 2: Adjusted for individual physical make-up and access to care } \\
\hline Women, colorectal & 1.49 & {$[1.32,1.69]$} & * & Women, breast & 2.17 & {$[1.90,2.47]$} & * \\
\hline Men, colorectal & 0.98 & {$[0.83,1.16]$} & & Women, colorectal & 3.23 & {$[2.77,3.77]$} & * \\
\hline Men, prostate & 0.46 & {$[0.40,0.53]$} & * & Men, colorectal & 2.12 & {$[1.77,2.55]$} & * \\
\hline \multicolumn{8}{|c|}{ Model 3: Adjusted for cancer type, individual physical make-up and access-to-care characteristics, health behaviors, and biological risk factors } \\
\hline Women, colorectal & 1.35 & {$[1.19,1.54]$} & * & Women, breast & 2.33 & {$[2.00,2.72]$} & * \\
\hline Men, colorectal & 0.92 & {$[0.78,1.10]$} & & Women, colorectal & 3.14 & {$[2.66,3.72]$} & * \\
\hline Men, prostate & 0.44 & {$[0.37,0.51]$} & * & Men, colorectal & 2.06 & {$[1.70,2.51]$} & * \\
\hline \multicolumn{8}{|c|}{$\begin{array}{l}\text { Model 4: Adjusted for cancer type, individual physical make-up and access-to-care characteristics, health behaviors, and biological risk factors, treatment } \\
\text { factors, community resources, geographical location, and year at cancer diagnosis }\end{array}$} \\
\hline Women, colorectal & 1.28 & {$[1.12,1.46]$} & * & Women, breast & 2.33 & {$[1.95,2.78]$} & * \\
\hline Men, colorectal & 0.88 & {$[0.73,1.05]$} & & Women, colorectal & 2.98 & {$[2.47,3.60]$} & * \\
\hline Men, prostate & 0.43 & {$[0.36,0.51]$} & * & Men, colorectal & 2.04 & {$[1.65,2.51]$} & * \\
\hline
\end{tabular}

Abbreviations: ARR, adjusted risk ratio; $R R$, risk ratio.

a Based on 53,821 elderly fee-for-service Medicare beneficiaries with incident breast, colorectal, and prostate cancers who were continuously enrolled in

Medicare Part A and B during the observation period and who were alive during the observation period. Asterisks represent significant differences in newly

diagnosed depression by cancer types, derived from complementary log-log regression.

${ }^{\mathrm{b}}$ Reference group $=$ women, breast.

${ }^{\mathrm{C}}$ Reference group $=$ men, prostate

${ }^{*} P<.001$.

available from published literature. ${ }^{5}$ The higher incidence of depression among women with breast cancer in our study may be due to differences in observation years, because it has been reported that the percentage of diagnosed depression among elderly Medicare beneficiaries increases over time. ${ }^{39}$ Additionally, although Danese et $\mathrm{al}^{5}$ used data from 1998-2002, the current study used data from $2007-$ 2012. We were not able to compare the incidence of depression for CRC and prostate cancer found in our study with other studies, because there are no published studies.

We found that the risk of newly diagnosed depression varied by cancer type. Women with CRC had a higher risk of newly diagnosed depression compared with those with breast cancer, even after adjustments for a comprehensive list of risk factors. Similarly, men with CRC had a higher risk of newly diagnosed depression compared with those with prostate cancer. As stated in the introduction, it is plausible that poor survival prognosis may have increased the risk of depression among CRC survi- vors. This study also found that women with CRC had a $52 \%$ higher risk of newly diagnosed depression compared with men with CRC. This finding confirmed sex differences in the risk of depression that have been documented previously. ${ }^{39}$ We also found in multivariable analyses that advanced stage at cancer diagnosis was associated with a higher risk of newly diagnosed depression compared with early stage at cancer diagnosis. This finding is consistent with prior published studies documenting that advanced stage at cancer diagnosis is associated with a high risk of depression. ${ }^{4,5}$

\section{Clinical Practice and Policy Implications}

Findings from our study have clinical practice and policy implications; they suggest that healthcare providers need to screen for depression, especially among individuals with CRC and those diagnosed with an advanced cancer stage. Because cancer is a dominant condition and most of the care is usually directed toward treating cancer, such screening is important to diagnose depression before it 
Alwhaibi et al

Table 4. Sex Differences in Newly Diagnosed Depression From Regression Analysis ${ }^{\mathrm{a}}$

\begin{tabular}{|lrrr|}
\hline Cancer Types $^{\mathrm{a}}$ & RR & $95 \% \mathrm{Cl}$ & $\begin{array}{c}\text { Significant } \\
\text { Differences }\end{array}$ \\
\hline Model 1: Adjusted for cancer types & & & \\
\hline Women, breast & 1.14 & {$[0.96,1.34]$} & \\
\hline Women, colorectal & 1.74 & {$[1.46,2.08]$} & $*$ \\
\hline Men, prostate & 0.47 & {$[0.40,0.57]$} & $*$ \\
\hline Cancer Types & ARR & $95 \%$ Cl & $\begin{array}{c}\text { Significant } \\
\text { Differences }\end{array}$ \\
\hline
\end{tabular}

Model 2: Adjusted for individual physical make-up and access-to-care characteristics

Women, breast $1.02 \quad[0.86,1.21]$

Women, colorectal $1.52 \quad[1.27,1.83]$

Men, prostate $0.47 \quad[0.39,0.57]$

Model 3: Adjusted for cancer type, individual physical make-up and access-to-care characteristics, health behaviors, and biological risk factors

Women, breast $1.13 \quad[0.95,1.35]$

Women, colorectal $1.52 \quad[1.27,1.83]$

$\begin{array}{lll}\text { Men, prostate } & 0.48 \quad[0.40,0.59]\end{array}$

Model 4: Adjusted for cancer type, individual physical make-up and access-to-care characteristics, health behaviors, and biological risk factors, treatment factors, community resources, geographical location, and year at cancer diagnosis

Women, breast $1.14 \quad[0.96,1.36]$

Women, colorectal $\quad 1.46 \quad[1.22,1.76]$

Men, prostate

$0.49[0.40,0.61]$

Abbreviations: ARR, adjusted risk ratio; RR, risk ratio.

aBased on 53,821 elderly fee-for-service Medicare beneficiaries with incident breast, colorectal, and prostate cancers who were continuously enrolled in Medicare Part A and B during the observation period and who were alive during the observation period. Asterisks represent significant differences in newly diagnosed depression by cancer types, derived from complementary log-log regression.

'Reference group = men, colorectal.

$\star P<.001$.

becomes severe. PCPs can play an important role in detection and treatment of depression among cancer survivors. A national survey of physicians conducted by the Cancer Care Outcomes Research \& Surveillance Consortium reported that $\mathrm{PCPs}$ are more involved in detection and treatment of depression in patients with cancer compared with oncologists ( $50 \%$ vs $18 \%$, respectively)..$^{40}$ Therefore, it is important that oncologists and PCPs work together to identify and treat depression among cancer survivors, because depression can negatively affect the health-related quality of life and survival after cancer diagnosis. ${ }^{9,11,27}$

Communication between oncologists and PCPs can be improved by the use of survivorship care plans, integrated collaborative care programs, use of electronic health records, and training PCPs in cancer care. ASCO considers survivorship care plans, which consist of a treatment summary and a follow-up plan, to be an essential component in survivorship care, because they can enhance care coordination and communication between oncologists, PCPs, and mental healthcare providers. ${ }^{41}$ One study reported that the communication of survivorship care plans to PCPs was associated with better care coordination. ${ }^{42}$

Collaborative care programs are one approach to integrating PCPs in cancer care to provide screening, monitoring, and treatment of depression. For example, the integrated collaborative care model, SMaRT Oncology-2 trial (Symptom Management Research Trial in Oncology-2), found that collaborative care between oncologists, PCPs, and trained nurses was associated with a significantly higher improvement in depression compared with the usual care. ${ }^{43}$ Communication between oncologists and PCPs can also be improved by the use of electronic health records and the training of PCPs in cancer care. It has been reported that use of electronic medical records and PCP training in cancer care improved communication and cancer care coordination between oncologists and PCPs. ${ }^{44,45}$

\section{Study Strengths and Limitations}

This study's findings need to be interpreted in the context of its advantages and limitations. One advantage is that this study used linked cancer registry and claims data, which allowed us to follow a large cohort of patients across a variety of health providers. Another advantage is that we also controlled for a comprehensive list of factors, such as cancer stage and treatment. This study also has some limitations. Because the study population was restricted to fee-for-service Medicare beneficiaries and those residing in SEER regions, the study findings are not generalizable to all Medicare beneficiaries. The focus of this study was on the most common types of cancer; future studies may need to investigate the risk of newly diagnosed depression and depression treatment rates among elderly patients with other types of cancer. It is plausible that depression may be underrecognized and we may have underestimated the rate of newly diagnosed depression. 
Risk of Depression and Cancer Type

\section{Conclusions}

This study has provided new evidence that there is variation in the risk of newly diagnosed depression by cancer type. Healthcare providers of cancer

\section{References}

1. Centers for Disease Control and Prevention. Basic Information for Cance Survivors. Available at: http://www.cdc.gov/cancer/survivorship/basic_info/ survivors/index.htm. Accessed December 5, 2015.

2. National Cancer Institute. Depression (PDQ) - Patient Version. Available at: http://www.cancer.gov/about-cancer/coping/feelings/depressionpdq\#section/_1. Accessed December 5, 2015.

3. National Cancer Institute. Adjustment to Cancer: Anxiety and Distress (PDQR) - Patient Version. Available at: http://www.cancer.gov/cancertopics/ pdq/supportivecare/adjustment/Patient/page1. Accessed December 5, 2015.

4. Chia VM, O'Malley CD, Danese MD, et al. Prevalence and incidence of comorbidities in elderly women with ovarian cancer. Gynecol Oncol 2013;129:346-352.

5. Danese MD, O'Malley C, Lindquist K, et al. An observational study of the prevalence and incidence of comorbid conditions in older women with breast cancer. Ann Oncol 2012;23:1756-1765.

6. Dalton SO, Laursen TM, Ross L, et al. Risk for hospitalization with depression after a cancer diagnosis: a nationwide, population-based study of cancer patient in Denmark from 1973 to 2003. J Clin Oncol 2009;27:1440-1445.

7. Fann JR, Thomas-Rich AM, Katon WJ, et al. Major depression after breast cancer: a review of epidemiology and treatment. Gen Hosp Psychiatry 2008;30:112-126.

8. Linden W, Vodermaier A, MacKenzie R, Greig D. Anxiety and depression after cancer diagnosis: prevalence rates by cancer type, gender, and age. J Affect Disord 2012;141:343-351

9. Rane PB. Burden of Colorectal Cancer Among the Elderly Medicare Beneficiaries in West Virginia: a Comparative Analysis With National Data [dissertation]. Morgantown: West Virginia University; 2014.

10. Kurtz ME, Kurtz JC, Stommel M, et al. Predictors of depressive symptomatology of geriatric patients with colorectal cancer: a longitudinal view. Support Care Cancer 2002;10:494-501.

11. Jayadevappa R, Malkowicz SB, Chhatre S, et al. The burden of depression in prostate cancer. Psychooncology 2012;21:1338-1345

12. Prasad SM, Eggener SE, Lipsitz SR, et al. Effect of depression on diagnosis, treatment, and mortality of men with clinically localized prostate cancer. J Clin Oncol 2014;32:2471-2478.

13. Krebber A, Buffart L, Kleijn G, et al. Prevalence of depression in cancer patients: a meta-analysis of diagnostic interviews and self-report instruments. Psychooncology 2014;23:121-130.

14. Weiss Wiesel TR, Nelson CJ, Tew WP, et al. The relationship between age, anxiety, and depression in older adults with cancer. Psychooncology 2014;24:712-717.

15. Cardoso G, Graca J, Klut C, et al. Depression and anxiety symptoms following cancer diagnosis: a cross-sectional study. Psychol Health Med 2016;21:562-570.

16. Reece JC, Chan YF, Herbert J, et al. Course of depression, mental health service utilization and treatment preferences in women receiving chemotherapy for breast cancer. Gen Hosp Psychiatry 2013;35:376-381.

17. Torres MA, Pace TW, Liu T, et al. Predictors of depression in breast cancer patients treated with radiation: role of prior chemotherapy and nuclear factor kappa B. Cancer 2013;119:1951-1959.

18. Hopwood P, Sumo G, Mills J, et al. The course of anxiety and depression over 5 years of follow-up and risk factors in women with early breast cancer: results from the UK Standardisation of Radiotherapy Trials (START). Breast 2010;19:84-91.

19. Korfage I, Essink-Bot $M$, Janssens $A$, et al. Anxiety and depression after prostate cancer diagnosis and treatment: 5-year follow-up. Br J Cancer 2006;94:10931098 .

20. Lee M, Jim HS, Fishman M, et al. Depressive symptomatology in men receiving androgen deprivation therapy for prostate cancer: a controlled comparison. Psychooncology 2014;24:472-477.

21. Sharp L, O'Leary E, Kinnear H, et al. Cancer-related symptoms predict psychological wellbeing among prostate cancer survivors: results from the PiCTure study. Psychooncology 2016;25:282-291. survivors may need to routinely screen individuals at high risk for depression, specifically those with CRC and those with an advanced stage of cancer at diagnosis.

22. Dinh KT, Reznor G, Muralidhar V, et al. Association of androgen deprivation therapy with depression in localized prostate cancer. J Clin Oncol 2016;34:1905-1912.

23. Vahdaninia M, Omidvari S, Montazeri A. What do predict anxiety and depression in breast cancer patients? A follow-up study. Soc Psychiatry Psychiatr Epidemiol 2010;45:355-361.

24. Brown LF, Kroenke K. Cancer-related fatigue and its associations with depression and anxiety: a systematic review. Psychosomatics 2009;50:440-447.

25. Canoui-Poitrine F, Reinald N, Laurent M, et al. Geriatric assessment findings independently associated with clinical depression in 1092 older patients with cancer: the ELCAPA cohort study. Psychooncology 2016;25:104-111.

26. DeSantis CE, Lin CC, Mariotto AB, et al. Cancer treatment and survivorship statistics, 2014. CA Cancer J Clin 2014;64:252-271.

27. Reyes-Gibby C, Anderson KO, Morrow P, et al. Depressive symptoms and health-related quality of life in breast cancer survivors. J Womens Health (Larchmt) 2012;21:311-318.

28. Jeffery DD, Linton A. The impact of depression as a cancer comorbidity: rates, health care utilization, and associated costs. Community Oncol 2012;9:216221.

29. Mausbach BT, Irwin SA. Depression and healthcare service utilization in patients with cancer [published online ahead of print April 21, 2016]. Psychooncology, doi: 10.1002/pon.4133.

30. Zheng Z, Yabroff KR, Guy GP Jr, et al. Annual medical expenditure and productivity loss among colorectal, female breast, and prostate cancer survivors in the united states. J Natl Cancer Inst 2015;108:10.1093/jnci/djv382.

31. Marmot M, Wilkinson R. Social Determinants of Health. Oxford, England: Oxford University Press; 2005.

32. National Cancer Institute. SEER Registry Groupings for Analyses. Available at: http://seer.cancer.gov/registries/terms.html. Accessed December 5, 2015.

33. Department of Health and Human Services. Area Health and Resources Files (AHRF). Available at: http://ahrf.hrsa.gov/overview.htm. Accessed December $5,2015$.

34. National Committee for Quality Assurance. The Healthcare Effectiveness Data and Information Set (HEDIS). Available at: http://www.ncqa.org/ HEDISQualityMeasurement.aspx. Accessed November 29, 2014.

35. Chronic Conditions Data Warehouse. CCW Chronic Conditions. Available at: https://www.ccwdata.org/web/guest/condition-categories. Accessed December 5, 2015.

36. Findley PA, Shen C, Sambamoorthi U. Depression treatment patterns among elderly with cancer. Depress Res Treat 2012;2012:676784.

37. Zhang AY, Cooper GS. Recognition of depression and anxiety among elderly colorectal cancer patients. Nurs Res Pract 2010;2010;693961.

38. Goodman RA. Defining and measuring chronic conditions: imperatives for research, policy, program, and practice. Preventing chronic disease 2013;10.

39. Akincigil A, Olfson M, Walkup JT, et al. Diagnosis and treatment of depression in older community-dwelling adults: 1992-2005. J Am Geriatr Soc 2011;59:1042-1051.

40. Klabunde $\mathrm{CN}$, Ambs A, Keating NL, et al. The role of primary care physicians in cancer care. J Gen Intern Med 2009;24:1029-1036.

41. Mayer DK, Nekhlyudov L, Snyder CF, et al. American Society of Clinical Oncology clinical expert statement on cancer survivorship care planning. J Oncol Pract 2014;10:345-351.

42. Forsythe LP, Parry C, Alfano CM, et al. Gaps in survivorship care plan delivery and potential benefits to survivorship care [abstract]. J Clin Oncol 2013;31(Suppl):Abstract 9594.

43. Sharpe $\mathrm{M}$, Walker $\mathrm{J}$, Hansen $\mathrm{CH}$, et al. Integrated collaborative care for comorbid major depression in patients with cancer (SMaRT oncology-2): a multicentre randomised controlled effectiveness trial. Lancet 2014;384:1099_ 1108 .

44. Klabunde $\mathrm{CN}$, Han $\mathrm{PK}$, Earle CC, et al. Physician roles in the cancer-related follow-up care of cancer survivors. Fam Med 2013;45:463-474.

45. Sada YH, Street RL Jr, Singh H, et al. Primary care and communication in shared cancer care: a qualitative study. Am J Manag Care 2011;17:259-265. 